\title{
LaNe RAGE: a new tool for genomic DNA flanking sequence determination
}

\author{
Daniel J. Park \\ Department of Zoology \\ The University of Melbourne \\ Gate 12, Royal Parade \\ Victoria 3010, Australia \\ Tel: 61383444351 \\ Fax: 61383447909 \\ E-mail: d.park@unimelb.edu.au
}

Financial support: This work was supported by the National Health and Medical Research Council of Australia grant no. 148630 to Professors J.A.M. Graves and M.B. Renfree, Associate Professor V.R. Harley and Dr. A.J. Pask.

Keywords: cloning, DNA fingerprinting, genomic DNA walking, PCR, sequencing, transposon mutagenesis.

Abbreviations: GAPDH: glyceraldehyde-3-phosphate dehydrogenase; gDNA: genomic DNA;

LaNe RAGE: lariat-dependent nested PCR for rapid amplification of genomic DNA ends;

PCR: polymerase chain reaction;

PGK1: phosphoglycerate kinase 1;

RAPD: randomly amplified polymorphic DNA.

The determination of genomic DNA sequence flanking a known region is often problematic. Existing technologies depend on multiple, efficient enzymecatalysed preparative processing steps and/or rely on relatively inefficient 'one-sided' PCR mechanisms. I demonstrate the application of a simple 'two-sided' PCR-based approach, lariat-dependent nested PCR for rapid amplification of genomic DNA ends (LaNe RAGE), applied to the mouse GAPDH and PGK1 gene flanking sequences. This demonstration offers great promise in applications such as genome walking, transposon mutagenesis mapping and DNA fingerprinting.

Current methods for PCR-based genomic DNA (gDNA) flanking sequence determination variously require relatively large amounts of starting material, efficient enzyme reaction steps reliant on restriction endonuclease, ligase and/or exonuclease or dependence on relatively inefficient 'one-sided' PCR reactions (Mueller and Wold, 1989; Arnold and Hodgson, 1991; Sarkar et al. 1993; Devon et al. 1995; Myrick and Gelbart, 2002). This study set out to demonstrate the feasibility of a new technology, LaNe RAGE, requiring only limited starting material without the need for involved pre-processing steps and capable of unleashing the exquisite sensitivity of fully gene-specific 'two-sided' nested PCR series (Figure 1).

\section{MATERIALS AND METHODS}

\section{gDNA extraction}

gDNA was extracted from the mouse HC11 mammary gland cell line using the DNeasy Tissue Kit (Qiagen, Clifton Hill, Vic., Australia).

\section{LaNe RAGE}

Primers were designed based on the GenBank accession sequences NT 039711.3 (BX469914.4) and NT 039206.3 (AL732526.8 reverse complement view) for PGK1 and $G A P D H$, respectively. Locations within these regions used for primer design are indicated after each primer sequence. $300 \mathrm{ng}$ gDNA was used as template in $50 \mu \mathrm{l}$ PfuTurbo ${ }^{\circledR}$ DNA Polymerase (Stratagene, Integrated Sciences, East Kew, Vic., Australia) -catalysed strand synthesis reactions primed by hybrid 5' gene-specific and 3' partially defined primers PGKN5CTCAC $(5$ 'GAGAATGCCAAGACTGGCCAAGCNNNNNCTCAC3': 26744-26766) or GAPN5CTCAC (5'TGGTCTACATGTTCCAGTATGACTCNNNNNCTCAC3': 3074-3098) for mouse PGK1 or GAPDH, respectively. The semi-random $3^{\prime}$ termini were chosen with the expectation that under the appropriate annealing conditions, they would bind at relatively frequent intervals adjacent to a known region. As such, a ladder of gene-specific products would be anticipated. The reaction mix tubes were heated to $94^{\circ} \mathrm{C}$ for $3 \mathrm{~min}$ and then placed on ice prior to addition of $1.5 \mu \mathrm{l} 10 \mathrm{mM}$ dNTPs and PfuTurbo ${ }^{\circledR}$ DNA Polymerase. The reaction tubes were then heated from $18^{\circ} \mathrm{C}$ to $72^{\circ} \mathrm{C}$ at a rate of $0.1^{\circ} \mathrm{C}$ per $5 \mathrm{sec}$ and held at $72^{\circ} \mathrm{C}$ for $10 \mathrm{~min}$ prior to placing on ice. Primers PGK1 (5'TTGGCACATCTCTGTATGATGAAG-3': 26622-26645) and PGK4 (5'-TACTGTGGCCTCTGGTATACCT-3': 26767-26788) or GAP1 (5'ATGGTGAAGGTCGGTGTGAACG-3': 2953-2974) and GAP4 (5'-CCGAGAATGGGAAGCTTGTCATC-3': 31313153), respectively, were added prior to thermocycling (36 
cycles of $94^{\circ} \mathrm{C}$ for $1 \mathrm{~min} ; 58^{\circ} \mathrm{C}$ for $1 \mathrm{~min} ; 72^{\circ} \mathrm{C}$ for $2 \mathrm{~min}$ ). The lariat structure formation and template generation depicted in the large boxed section of Figure 1 occurs inherently during the thermal cycling of LaNe RAGE.

$1 \mu \mathrm{l}$ product from each first round PCR was included as template in $50 \mu \mathrm{l}$ hot-start second round PCRs employing primers PGK2

GGAGCCAAGATTGTCAAAGATCTC-3': 26648-26671) and PGK5 (5'-CTGTTCGCTTATGGAAGGGATC-3': 26814-26835) or GAP2 (5'CAGTGGCAAAGTGGAGATTGTTGC-3': $\quad$ 3018-3041) and GAP5 (5'-GGAGCGAGACCCCACTAAC-3': 31803198), respectively, and PfuTurbo ${ }^{\circledR}$ DNA Polymerase (40 cycles of $94^{\circ} \mathrm{C}$ for $1 \mathrm{~min} ; 60^{\circ} \mathrm{C}$ for $1 \mathrm{~min} ; 72^{\circ} \mathrm{C}$ for $2 \mathrm{~min}$ ). Products were visualized by UV trans-illumination after agarose gel electrophoresis.

\section{DNA sequencing}

Major PCR products were gel extracted using the QIAEX ${ }^{\circledR I I}$ bulk silica DNA purification system (Qiagen) and subjected to sequencing employing $\mathrm{ABI}$ PRISM®BigDye ${ }^{\mathrm{TM}}$ Terminators v.3.1 (Applied Biosystems, Scoresby, Vic., Australia) primed by PGK2, PGK5, GAP2 or GAP5. Electrophoresis was performed by the Wellcome Trust Sequencing Facility, a facility of Monash Institute of Reproduction and Development and Prince Henry's Institute of Medical Research, Monash Medical Centre, Clayton, Vic., Australia.

\section{RESULTS AND DISCUSSION}

Figure 2 illustrates the agarose gel profiles resulting from application of LaNe RAGE to the mouse GAPDH and $P G K 1$ genes. The $280 \mathrm{bp}$ and $580 \mathrm{bp}$ products for GAPDH and $P G K 1$, respectively, as the major products, were chosen for DNA sequence analysis (Figure 3 and Figure 4). Nucleotide-nucleotide BLAST searching and Clustal W alignment analysis, indicated that they represented GAPDH and PGK1 flanking regions, respectively (Figure 5 and Figure 6). Furthermore, the presence of gene specific hybrid primer-derived sequence confirmed that the products had been yielded via a LaNe RAGE mechanism. Major bands were consistently observed in independent experiments and were confirmed to be gene-specific by DNA sequence analysis. The GAPDH profile exhibits an expected ladder of products while that for PGK1 shows a major product and relatively few minor distinct bands. It is possible that the gene-specific region of the PGKN5CTCAC primer contributed to enhance binding at a particular site, such that the resulting major product outcompeted others in the ensuing PCRs. The background smear observed in the GAPDH product could be due to primer binding at more regular intervals than anticipated (for example, TCAC or CAC sites). Altered primer design and annealing conditions change product profiles to suit the requirements of the application. For example, the design of 'less random' primers for use in the initial annealing step should result in a ladder with longer products and bigger 'steps'. Nonetheless, the described experiments effectively serve to demonstrate the potential of LaNe RAGE.

This demonstration of a new simple and effective tool for derivation of gDNA flanking sequence requiring only limited starting material has implications not only for genome walking, but also for identification of insertion sites resulting from transposon mutagenesis screens and for DNA fingerprinting applied in contexts such as pathogenic bacteria strain typing. These applications should simply require optimization of primer design and thermocycling conditions. LaNe RAGE should offer greater specificity and sensitivity than existing approaches via a quick, simple and robust mechanism. At the heart of this is the capacity for 'two-sided' gene-specific nested PCR series without the requirement for involved enzyme pre-processing steps.

\section{REFERENCES}

ARNOLD, C. and HODGSON, I.J. Vectorette PCR: a novel approach to genomic walking. PCR Methods and Applications, 1991, vol. 1, no. 1, p. 39-42.

DEVON, R.S.; PORTEOUS, D.J. and BROOKES, A.J. Splinkerettes - improved vectorettes for greater efficiency in PCR walking. Nucleic Acids Research, May 1995, vol. 23, no. 9, p. 1644-1645.

MUELLER, Paul R. and WOLD, Barbara. In vivo footprinting of a muscle specific enhancer by ligation mediated PCR. Science, November 1989, vol. 246, no. 4931, p. 780-786.

MYRICK, Kyl V. and GELBART, William M. Universal fast walking for direct and versatile determination of flanking sequence. Gene, February 2002, vol. 284, p. 125131.

SARKAR, G.; TURNER, R.T. and BOLANDER, M.E. Restriction site PCR: a direct method of unknown sequence retrieval adjacent to a known locus by using universal primers. PCR Methods and Applications, 1993, vol. 2, no. 4, p. 318-322. 


\title{
APPENDIX
}

\author{
Figures
}

\section{LaNe RAGE reaction series}
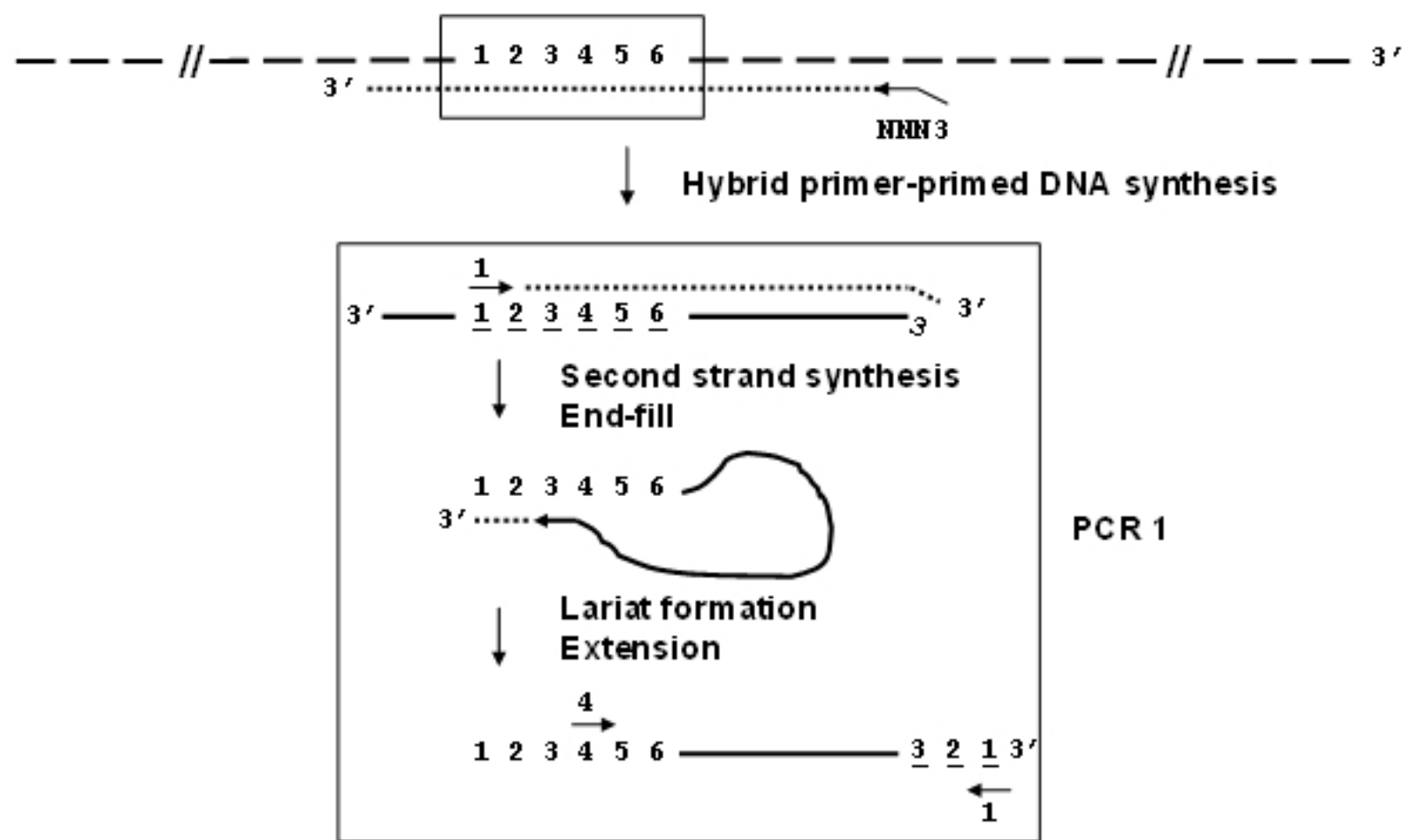

\section{PCR 1}

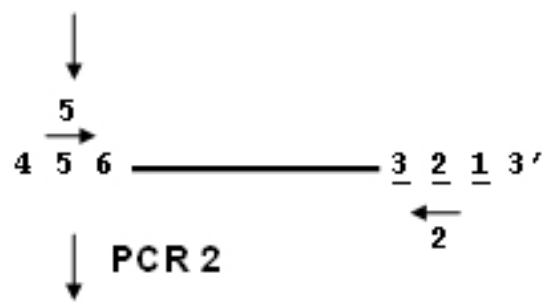

56

$\underline{3} \underline{2}^{3}$

Figure 1. Schematic representation of LaNe RAGE. Dashed lines represent gDNA. Dotted lines represent DNA polymerization. Solid lines represent DNA formed during the previous step. $3^{\prime}$ indicates the $3^{\prime}$ end of DNA strands. The small box represents a known region of gDNA. The large box indicates intrinsic steps which take place during the first PCR of the LaNe RAGE procedure. Numbers 1 to 5 indicate genespecific primer binding sites within a known region of gDNA. Underlined numbers indicate reverse complementary sequence relative to the initial gDNA strand. Number 6 indicates an optional gene-specific primer binding site for direct sequencing of product. Arrows indicate sites of primer binding. NNN3 labels the special hybrid primer used to prime initial DNA synthesis, consisting of a 5' gene-specific sequence (corresponding to position number 3 ) adjacent to 3 ' terminal degenerate, part-defined or defined sequence.

Initially, the NNN3 hybrid primer binds in a semi-random fashion at regular intervals along genomic DNA to prime DNA synthesis. During thermal cycling, gene-specific primer 1 binds at a specific site within the known region of the product of NNN3-primed extension to prime another extension reaction. This results in a product with gene-specific sequence at its $5^{\prime}$ and $3^{\prime}$ (corresponding to gene-specific sequence introduced by the hybrid primer) ends, with unknown sequence between. During thermal cycling, this is able to form a lariat structure, which primes intra-strand extension. The resultant product possesses $5^{\prime}$ gene-specific sequence and extended 3 ' gene-specific sequence with unknown sequence between, which is amenable to two-sided gene-specific PCR series. 
Park, D. J.

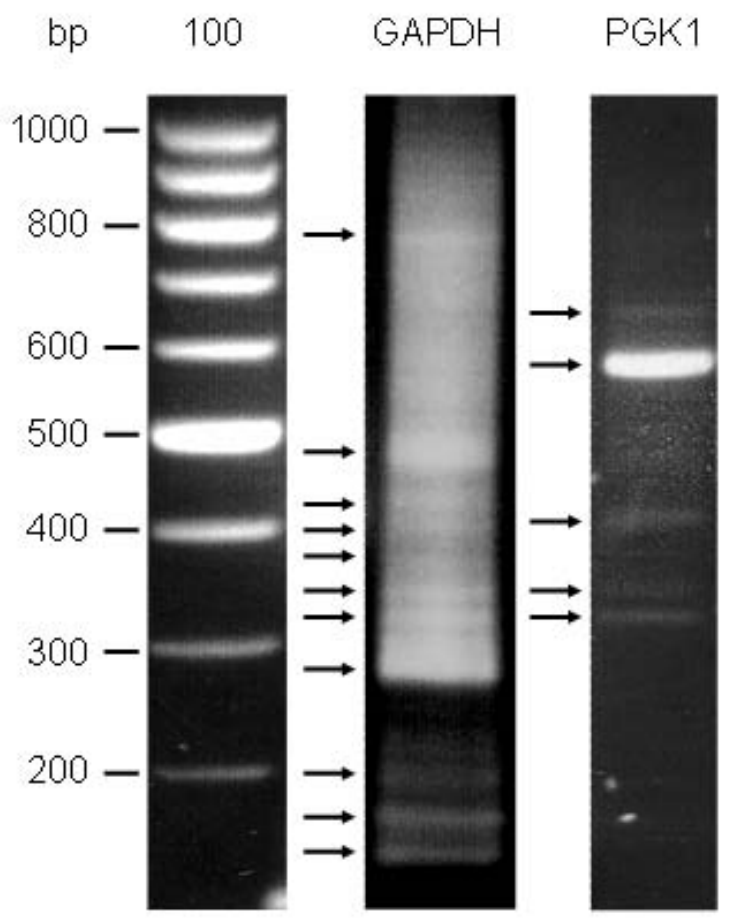

Figure 2. Separation on 1.4\% agarose 0.5xTBE agarose gel of products yielded from LaNe RAGE applied to mouse GAPDH and PGK1 genes. 100 indicates Promega 100 bp ladder (Promega Corporation, Annandale, NSW, Australia). Arrows indicate discrete product bands. The major products (280 bp and $580 \mathrm{bp}$ for GAPDH and PGK1, respectively) were selected for sequence analysis. 
Known sequence between GAP2 and GAPN5CTCAC (sense orientation with respect to the reverse comple ment display of

\section{AL732526.8)}
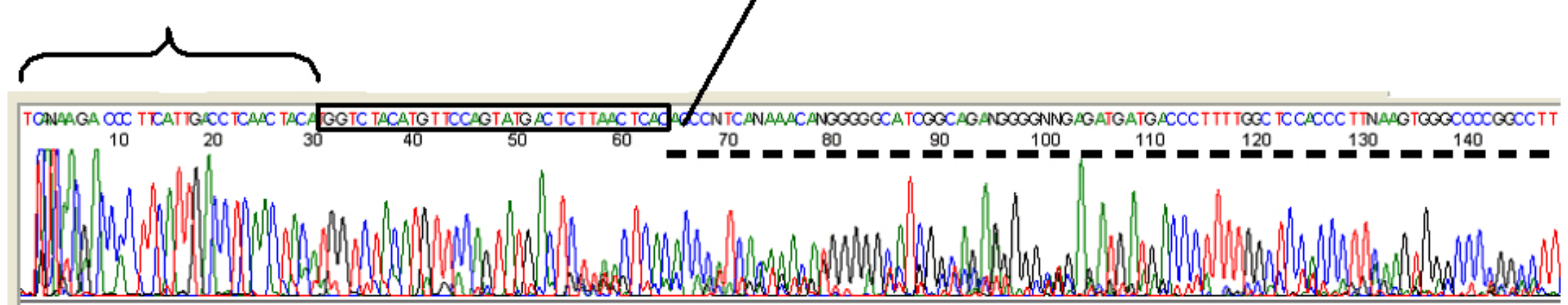

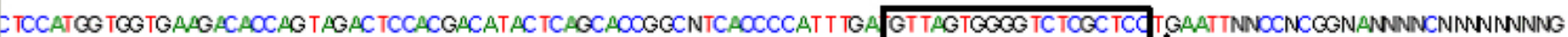

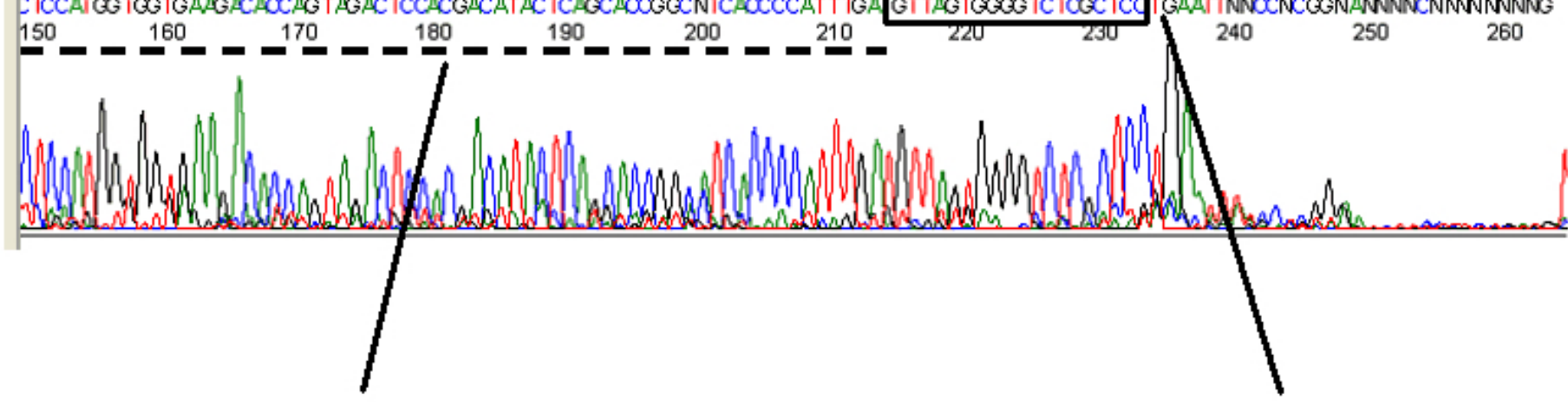

'Unknown region' in anti-sense

orientation: corresponds to $3348-3199$ of

the reverse complement display of

AL732526.8)

Figure 3. Chromas generated chromatogram depicting sequence for the LaNe RAGE-derived 280 bp major GAPDH product. The sequencing reaction was primed using GAP2.

Dashed lines represent 'unknown' sequence. Boxed regions represent sequence corresponding to primers. The product is consistent with the mechanism shown in Figure 1.
GAPN5CTCAC (primer sequence in sense

(ation: gene-specific region

complement display of AL732526.8) 
Known sequence between PGK2 and PGKN5CTCAC (sense orientation with respect to the sense display of BX469914.4)
PGKN5CTCAC (primer sequence in sense orientation: gene-s pecific region corresponds to $26744-26766$ of the sense display of BX469914.4)

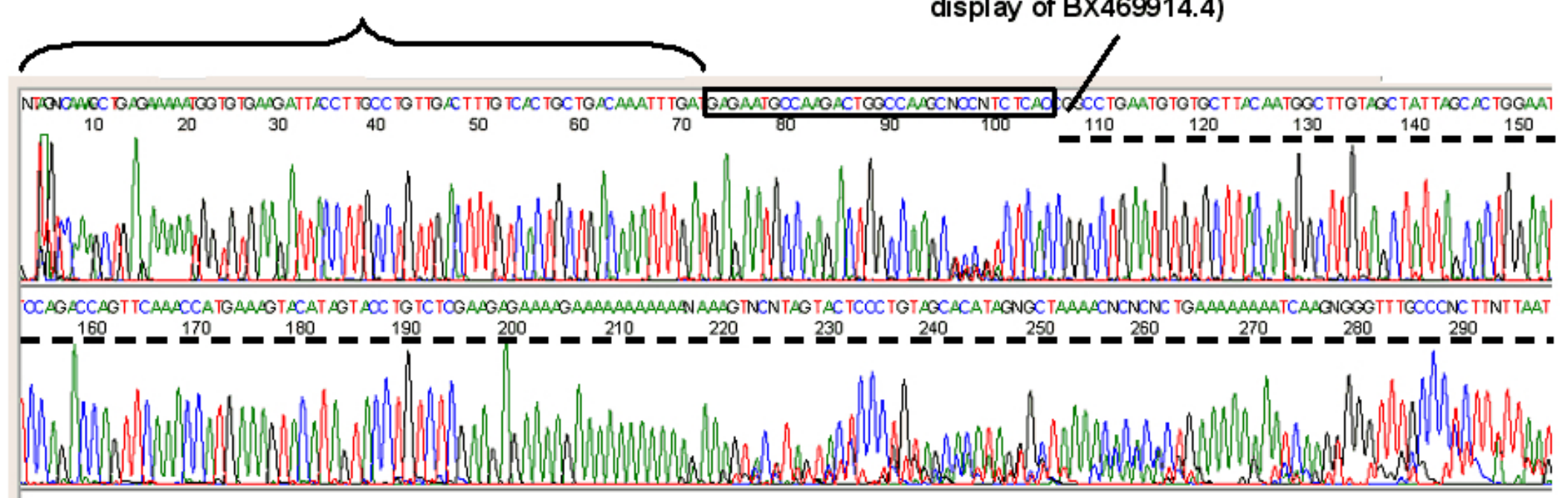

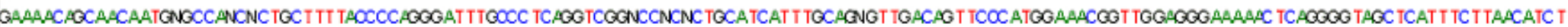

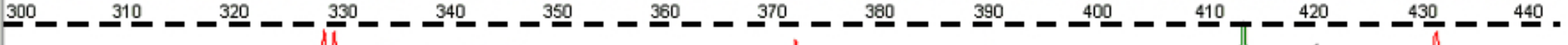

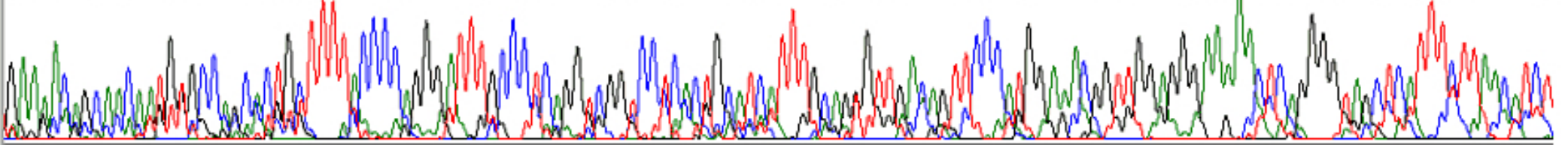

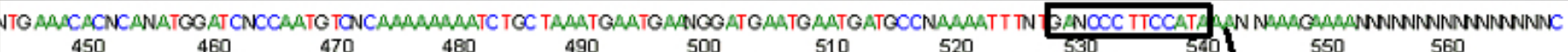

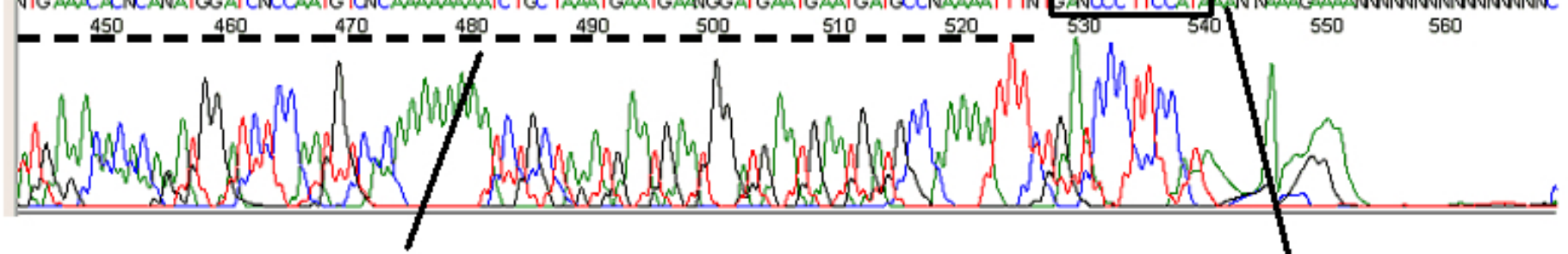

'Unknown region' in anti-sense orientation: corresponds to $27257-26836$ of the sense display of BX469914.4)
PGK5 (primer sequence in anti-sense orientation : corresponds to $26835-26823$ of the sense display of BX469914.4)

Figure 4. Chromas generated chromatogram depicting sequence for the LaNe RAGE-derived $\mathbf{5 8 0}$ bp major PGK1 product. The sequencing reaction was primed using PGK2. Dashed lines represent 'unknown' sequence. Boxed regions represent sequence corresponding to primers. The product is consistent with the mechanism shown in Figure 1. 
G280-2re

AI732526. 8

G280-2re

AL732526.8

G280-2rC

AI 732526.8

G280-2re

AI732526. 8

G280-2rC

AI732526.8
ACAGTCAAGGCCGAGAATGGGAAGCTTGTCATCAACGGGAAGCCCATCACCATCTTCCAG

GAGCGAGACCCCACTAACATCAAATGGGGTGAGGCCGGTGCTGAGTATGTCGTGGAGTCT

ACTGGTGTCTTCACCACCATGGAGAAGGCCGGGGCCCACTTNAAGGGTGGAGCCAAAAGG 102 ACTGGTGTCTTCACCACCATGGAGAAGGCCGGGGCCCACTTGAAGGGTGGAGCCAAACGG 3300 $* * * * * * * * * * * * * * * * * * * * * * * * * * * * * * * * * * * * * * * * * * * * * * * * * * * * * * * * * * 1$
GTCATCATCTCNNCCCCNTCTGCCGATGCCCCCNTGTTTNTGANGGGT- GTCATCATCTCOGCCCCTTCTGCCGATGCCCCCATGTTTGTGATGGGTGTGAACCACGHG * CATCATCTCCGCCCCTTCTGCCGATGCCCCCATGTTTGTGATGGGTGTGACCACGAG 3360 $* * * * * * * * * * * \quad * * * * * * * * * * * * * * * * * * * * * * * * * * * * * * * *$

AAATATGACAACTCACTCAAGATTGTCAGCAATGCATCCTGCACCACCAACTGCTTAGCC 3420

Figure 5. Clustal W generated alignment of the 'unknown region' of the GAPDH LaNe RAGE product (reverse complement of that depicted in Figure 3) with the reverse complement view of the corresponding GAPDH genomic segment AL732526.8. Numbers at the ends of the AL732526.8 lines indicate the base positions within the complete genomic segment. 


$$
\begin{aligned}
& \text { P580-2rc } \\
& \text { BX } 469914,4 \\
& \text { CCAAGCTACTGTGGCCTCTGGTATACCTGCTGGCTGGATGGTGAGTCAGCTGGCTGTTCG } 26820 \\
& \text { P580-2re } \\
& \text { BX469914.4 } \\
& \text {-ANAAATTTTHGGCATCATTCATTCATCCNTTCATTCATTTAGCAG } 45 \\
& \text { CTTATGGAAGGGATCAGAAATTTTTGGCATCATTCATTCATCCATTCATTCATCTAGCAG } 26880 \\
& *^{* * * * * * * *} * * * * * * * * * * * * * * * * * * * * * * * * * * * * * * * * * * * \\
& \text { P580-2ro } \\
& \text { BX469914. } 4 \\
& \text { P580-2ro } \\
& \text { BX469914.4 } \\
& \text { P580-2re } \\
& \text { BX469914. } 4 \\
& \text { P580-2re } \\
& \text { BX469914. } 4 \\
& \text { P580-2rC } \\
& \text { BX469914, } 4 \\
& \text { P580-2rC } \\
& \text { BX469914. } 4 \\
& \text { P580-2rc } \\
& \text { BX469914, } 4 \\
& \text { P580-2rC } \\
& \text { BX469914, } 4
\end{aligned}
$$

